\title{
The Chemical Investigation of Papaver Seeds
}

\author{
Hannis Fadzillah Mohsin, Ibtisam Abdul Wahab, Nadia Idiana Md Nasir, Nur Hidayah Zulkefli \& \\ Nurul Intan Shafinas Md Nasir \\ Faculty of Pharmacy, Universiti Teknologi MARA, 42300 Puncak Alam, Selangor Darul Ehsan, Malaysia \\ E-mail: \{hannis,ibtisam\}@puncakalam.uitm.edu.my,nadiaidiana@gmail.com, \\ \{hidayah_sakuracsgppkls,shafinas_aurora85\}@yahoo.com
}

\begin{abstract}
The Papaver species (common name: poppy; plant family: Papaveraceae) has been involved in a wide array of research, particularly in the interest of botany, food biotechnology and clinical chemistry. Its bright and colourful flowers receive international recognition in floriculture industry, while the seeds' function was recently reviewed as a source of linoleic acid and other functional food. However, abuse cases were also reported, due to the presence of natural opiates, such as heroine, morphine and codeine, thus contributing to the opium dependence. Here, the results from a preliminary test concerning two samples are presented. The experiment consisted of Papaver seeds from a local bakery and the herbalist. Following a series of organic extraction, three reagents including Mayer, Draggendorf and Bouchardat solutions were utilized in order to screen the phytochemical constituents. The detection method respectively gave positive results of white, orange and brown reddish precipitates, indicating the occurrence of alkaloids. The most significant precipitations were revealed from the chloroform extract of the herbal example. Earlier separation procedure utilizing hexane was withdrawn; due to the deficiency of the extractive. Chromatographic technique could be applied in order to separate the major compounds. Hopefully, this scientific investigation could provide educational information covering the topics of recreational drugs and essential pharmaceutical materials.
\end{abstract}

Keywords-Papaver, seed, chemistry

\section{INTRODUCTION}

The Papaver species (common name: poppy; plant family: Papaveraceae) has been involved in a wide array of research, particularly in the interest of botany [1], food biotechnology [2] and clinical chemistry [3]. Its bright and colourful flowers receive international recognition in floriculture industry. Meanwhile, the seeds' function was recently reviewed as a source of functional food such as linoleic acid (Fig. 1) [4], a compound that could possibly reduce the risk of heart diseases.

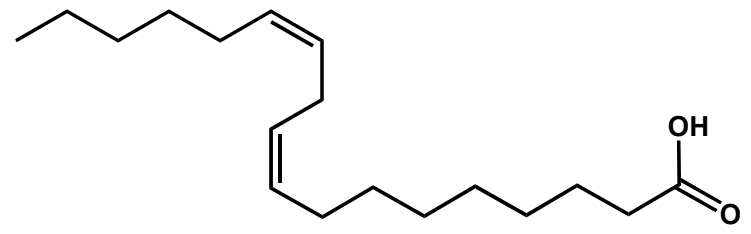

Fig. 1. Linoleic acid.

In addition, the Papaver seeds are popular substances in modern pastry and confectionary business, such as in instant cake mixtures and tea preparations. This is owing to their attractive, pleasant blue to purplish hues and their crunchiness taste. However, abuse cases were also reported [5], due to the presence of opiates. These natural alkaloidal drugs such as morphine and codeine, which provide stimulating and pain relieving effects (Fig. 2), could contribute to the opium dependence [6]. They are amines and have basic property. They can form salts with many acids; the most commonly found are phosphate and sulphate salts [7].

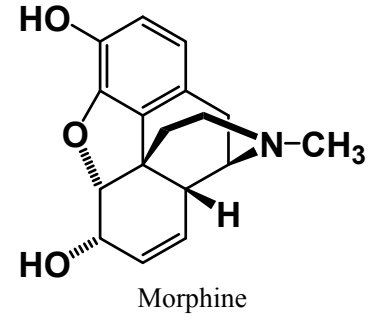

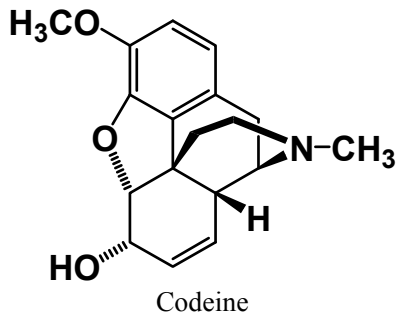

Fig. 2. The natural alkaloidal drugs in Papaver species.

The salt forms are water soluble but the free alkaloids generally are not. For example, thebaine hydrochloride is more water soluble than free thebaine. Acidic and neutral free alkaloids, such as caffeine in Coffee species (family: Rubiaceae), are hydrophilic and may not form salts (Fig. 3). 


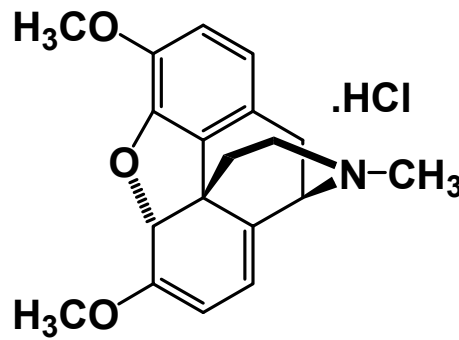

Thebaine hydrochloride

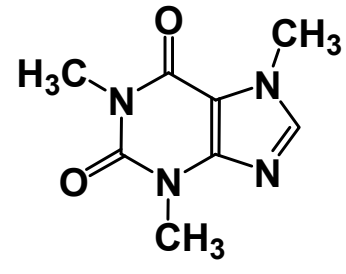

Caffeine
Fig. 3. The structure of thebaine hydrochloride and caffeine.

Plant alkaloids can be variably bioavailable. They can be irreversibly bound and precipitated by tannins, making them almost totally unabsorbable, thus unextractable due to high complexity [8]. The molecular weight of the bound Papaver alkaloids seemed to increase during ripening [8]. It is suggested that these bound substances represent transitional forms in the metabolism and translocation of morphine from latex to seed [8]. In this paper, the results from a preliminary chemical test concerning two Papaver samples are presented. The discussion will also cover the suitable solvent systems for the extraction, as well as the solubility of the extracts.

\section{METHODOLOGY}

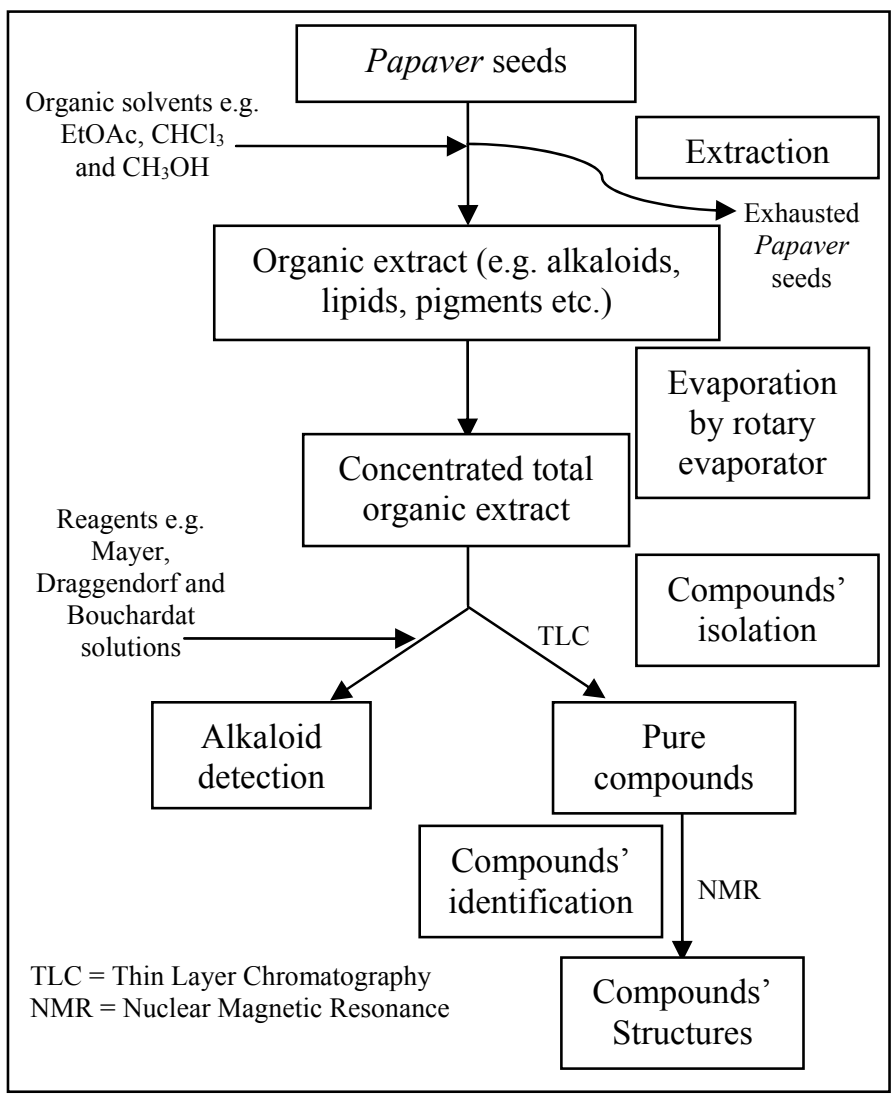

Fig. 3. The proposed research methodology.

The current and proposed research methodology could be clearly described by a flow chart, as shown in Fig. 3. The experiment consisted of the seeds or blue maw, from a local bakery and European herbalist, obtained from the dark red opium poppy of Papaver species. Following a series of organic extraction [triplicate maceration of ethyl acetate (EtOAc), chloroform $\left(\mathrm{CHCl}_{3}\right)$ and methanol $\left(\mathrm{CH}_{3} \mathrm{OH}\right)$, simultaneously) at room temperature, three reagents including Mayer, Draggendorf and Bouchardat solutions were utilized to monitor the phytochemical constituents. This screening procedure was adapted from the standard pharmacognostical assessment of crude plant materials.

\section{RESULTS \& DISCUSSION}

The latest horticultural review of opium poppy, one of the earliest domesticated plant species, was printed [9]. This particular opium poppy or species of Papaver somniferum, provides the latex which is a commercial source of the analgesics, morphine and codeine [10]. The review covered the Papaver's cultivation practices, infraspecific systematics, operations during developmental periods, ripening and harvest, utilization in medicinal, culinary (oil and spice) and bakery, plus the chemical diversity of Papaver's European and Asiatic cultivars. The consumption of whole poppy seeds could still be seen for glazing cakes, croissants and milk loaves [9]. Meanwhile, morphine, codeine and thebaine were reported as the major alkaloids of Papaver somniferum [11]. The chemical structures of opium alkaloids and related important compounds from Papaver seeds were available. Some structures were shown in Fig. 1 and 2 and presented in [7]. The alkaloid content of opium is approximately $10-20$ percent with more than 40 individual alkaloids have been isolated [7].

Generally, the reductions of small particle sizes that could be achieved by crushing, grinding or milling would increase the available surface area and the volumetric yields for the plant extraction, as commented [12]. In this study, the initial step is to determine the alkaloidal content from the Papaver seeds, qualitatively. Therefore, the powderisation and pelletization were not performed, since the particle sizes of the seeds were not the major concern. Furthermore, the quantitative measurement is not the main scope of this work. In an analysis, the optimal sample preparation was achieved by using unground poppy seeds [13].

Previous analyses on the extraction of plants' alkaloids were also referred. Earlier, the residue from the dried latex of Papaver orientale and Papaver pseudo-orientale capsules were chromatographed by using mixtures of ethyl acetate-methanol-ammonia [14]. Next, the alkaloidal content of the coca leaf (Erythroxylum coca, family: Erythroxylaceae) in coca tea bags was determined by two different extraction methods; methanolic exhaustive Soxhlet extraction and methanolic mechanical agitation [15]. This is followed by a report from Popa and co-workers [16] where morphine and codeine were extracted from Papaver somniferum capsules with chloroform:isopropanol $(3: 1, \mathrm{v} / \mathrm{v})$ in basic condition.

In this study, a former separation procedure involved the hexane solution. Nevertheless, this approach was withdrawn, due to the deficiency of the extractive. This is parallel with [17], where hexane was used for removing Papaver lipids. After concurrent extraction with ethyl acetate, chloroform and methanol, it was observed that the seeds' colour was changed to dark brown (Fig. 4). Therefore, it was believed that the extraction could occur in these conditions. The 
choice of these organic solutions for extraction could be justified by referring to their relative polarity index, where methanol, ethyl acetate and chloroform could be classified as high, medium and low polarity solvents, respectively.

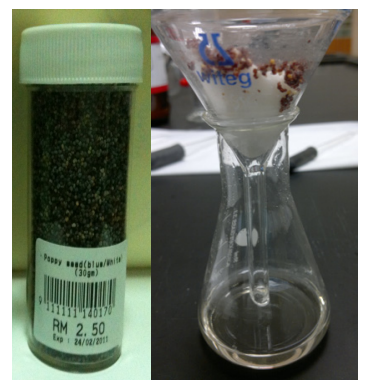

Fig. 4. The seeds' colour before (left) and after (right) the extraction.

Later, the detection method using Mayer, Draggendorf and Bouchardat solutions respectively gave white, orange and brown reddish precipitates. This observation could suggest the occurrence of alkaloids in the samples (as shown in Fig. 5). The precipitations were also revealed from the chloroform extract of the herbal example. It was mentioned that the plant could lose its opium content, once the seed formation occurs. Nevertheless, this preliminary result would be in contrast with the product's statement.

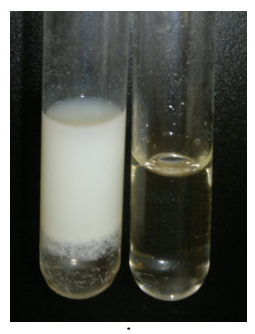

i

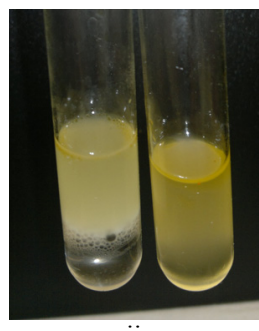

ii

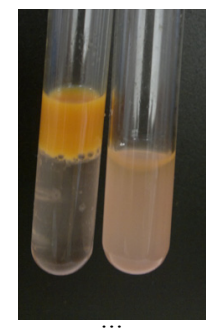

iii
Fig. 5. The precipitates occurred in the respective reagent $(i=$ Mayer, $i i=$ Draggendorf, iii = Bouchardat) in the chloroform extract. The herbalist's sample was in the left glass tube, whereas the bakery's seed was in the right glass tube.

Fig. 5 also shows that the herbalist's sample on the left hand side contained immiscible liquids, whereas the bakery's sample on the right hand side was not. These two different phenomena could be explained by the types of alkaloid in the research specimen. As a result of the free alkaloid diffusion in Bouchardat reagent (a potassium iodide-iodine solution) in Fig. 5 (iii), the mixtures appeared as miscible. Therefore, it is believed that herbalist's organic extract consists of complexes of bound alkaloids. This is due to their insolubility in the aqueous reagent mixtures.

In this test, normal extractions were applied, in contrast to an earlier publication [18]. A vigorous acid hydrolysis of Papaver seeds could lead to the production of alkaloid-like substances, including codeine. The authors also suggested that morphine is rapidly metabolised in the latex into both alkaloid- and non-alkaloidal compounds which were ethanol insoluble and bound forms. The bound forms are stored in the seeds and broken down into smaller alkaloid-like substances during germination [18]. Therefore, the contrast in the herbal product's claim could also due to the presence of this degradation of bound forms.

Due to the raised issue on the presence of opiate alkaloids in poppy seed intended for use in food, an efficient method for routine analysis of morphine and codeine using liquid chromatography in combination with tandem mass spectrometry on a triple quadrupole instrument (LC/MS/MS) was developed [13]. The optimal sample preparation was found to be an hour of cold centrifugal extraction of unground poppy seed with $0.1 \%$ acidic methanol. Afterwards, the fate of morphine during food processing was studied. Their experiments led to a significant reduction of morphine and codeine. For the poppy cake, a maximum of half morphinoid content was recovered. Meanwhile in poppy buns, only $3 \%$ of the original morphine content was found at the highest temperature $\left(220{ }^{\circ} \mathrm{C}\right)$. Ground poppy seed showed significantly lower recoveries than untreated seed. It was suggested that morphine elimination during food processing has to be taken into account to monitor its limit in poppy seed [13]. Currently, latest food science investigations manage to include innovative design of meat product in replacing animal fat with ground poppy seed paste, as an effective method to significantly decrease the cholesterol content of burgers [2].

\section{CONCLUSIONS}

The main finding of this work could be concluded. From the literature review, the domestic applications of the Papaver seeds could be mostly observed in culinary practices, such as salad dressings and muffin mixtures. It could be suggested that the bakery's poppy might have gone through the alkaloid removal process, due to less significant free alkaloid precipitation occurred in the detection procedure. In another thought, the bakery's supply could be originated from a specific Papaver species, for example, Papaver bracteatum, a close relative to Papaver somniferum. This is because Papaver bracteatum contains principally about $3 \%$ thebaine, with only traces of codeine and no morphine [7]. The author hoped that $P$. bracteatum could replace $P$. somniferum as a source of codeine, in order to lessen the demand for growth of the latter. Previous report supported $P$. bracteatum, a native to Iran and southern Russia, having the potential as a viable material for a commercial source of thebaine [19]. This review also included the possible chemical conversion of thebaine into codeine, economic and social advantages of growing $P$. bracteatum over opium poppy ( $P$. somniferum), plus the value of $P$. bracteatum seed oil for cooking and industrial use. For an example, linoleic acid or cis,cis-9,12octadecadienoic acid (Fig. 1) was reported as the key component of the poppy seed oil of the Turkish origin, which would possibly contribute to reduced risk of heart diseases.

Finally, this study has highlighted the key points where educational information that covers the topics of recreational alkaloidal drugs and essential pharmaceutical materials could be provided via scientific investigations. Future work would include advanced chromatographic techniques, in order to separate the major compounds. 


\section{ACKNOWLEDGMENT}

We would like to thank Universiti Teknologi MARA for the support.

\section{REFERENCES}

[1] H. S. Acharya and V. Sharma. (2009) Molecular Characterization of Opium Poppy (Papaver somniferum) Germplasm. American Journal of Infectious Diseases, 5 (2): 155-160.

[2] V. Gök, L. Akkaya, E. Obuz and S. Bulut. (2011) Effect of ground poppy seed as a fat replacer on meat burgers. Meat Sci. (in print). Retrieved online on $1^{\text {st }}$ July 2011.

[3] K. Jankovicová, P. Ulbrich and M. Fuknová. (2009) Effect of poppy seed consummation on the positive results of opiates screening in biological samples. Leg. Med. (Tokyo). 2009 Apr; 11, S, S416 - 418.

[4] E. Small. (2004) Narcotic plants as sources of medicinals, nutraceuticals and functional foods. In: Hou, F. F., Lin, H. S., Chou, M. H., Chang, T. W. (eds). 2004. Proceedings of the International Symposium on the Development of Medicinal Plants, Hualien District Agricultural Research \& Extension Station,Taiwan.pp.11-66.

[5] K. Brayer, T. Harwood, R. Inder, R. Beasley and G. Robinson. (2007) Poppy seed tea and opiate abuse in New Zealand. Drug and Alcohol Review, 26, 215-219.

[6] S. B. Nanjayya, P. Murthy, P. K. Chand, A. Kandaswamy, B. S. Nikketha, V. Benegal and M. Shivappa. (2009) A case of poppy tea dependence in an octogenarian lady. Drug and Alcohol Review, 1-3.

[7] P. L. Schiff, Jr. (2002) Opium and Its Alkaloids. Am. J. Pharm. Educ, 66, 186-194.

[8] J. W. Fairbairn and M. J. Steele. (1980) Bound forms of alkaloids in Papaver somniferum and P. bracteatum. Phytochemistry, 19 (11), 2317-2321.

[9] P. Tétényi. (2010) Opium Poppy (Papaver somniferum): Botany and Horticulture, in Horticultural Reviews, Volume 19 (ed J. Janick), John Wiley \& Sons, Inc., Oxford, UK
[10] N. Vijaya Sree, P. Udayasri, V. V. Aswani kumar Y., B. Ravi Babu, Y. Phani kumar and M. Vijay Varma. (2010) Advancements in the Production of Secondary Metabolites. Journal of Natural Products, 3, 112-123.

[11] V. La Valva, S. Sabato and G. Siniscalco Gigliano. (1985) Morphology and Alkaloid Chemistry of Papaver setigerum DC. (Papaveraceae). Taxon, 34 (2), 191-196.

[12] E. L. Chin Cheah, L. Wah Chan and P. W. Sia Heng. (2006) Supercritical carbon dioxide and its application in the extraction of active principles from plant materials. Journal of Pharmaceutical Sciences I, $59-71$

[13] C. Sproll, R. C. Perz and D. W. Lachenmeier. (2006) Optimized LC/MS/MS Analysis of Morphine and Codeine in Poppy Seed and Evaluation of Their Fate during Food Processing as a Basis for Risk Analysis. J. Agric. Food Chem., 54 (15), 5292-5298.

[14] A. Shafiee, I. Lalezari, P. Nasseri-Nouri and R. Asgharian. (1975) Alkaloids of Papaver orientale and Papaver pseudo-orientale. Journal of Pharmaceutical Sciences, 64 (9), 1570-1572.

[15] A. J. Jenkins, T. Llosa, I. Montoya and E. J. Cone. (1996) Identification and quantitation of alkaloids in coca tea. Forensic Science International, 77 (3), 179-189.

[16] D.-S. Popa, R. Oprean, E. Curea and N. Preda. (1998) TLC-UV densitometric and GC-MSD methods for simultaneous quantification of morphine and codeine in poppy capsules. Journal of Pharmaceutical and Biomedical Analysis, 18 (4-5), 645-650.

[17] M. D. Grove, G. F. Spencer, M. V. Wakeman and H. L. Tookey. (1976) Morphine and codeine in poppy seed. J. Agric. Food Chem., 24 (4), 896-896.

[18] J. W. Fairbairn and S. El-Masry. (1968) The alkaloids of Papaver somniferum L.-VI.: "Bound" morphine and seed development. Phytochemistry, 7 (2), 181-187.

[19] M. Seddigh, G. D. Jolliff, W. Calhoun and J. M. Crane. (1982) Papaver bracteatum, Potential Commercial Source of Codeine. Economic Botany, 36 (4), 433-441. 